\title{
Leaders
}

\section{Continuing professional development: some new perspectives}

\author{
Clair du Boulay
}

Continuing professional development (CPD) is a process of lifelong learning for all individuals and teams, aimed at meeting the needs of patients and delivering the health outcomes and healthcare priorities of the NHS, while enabling professionals to expand and fulfil their potential. ${ }^{1}$ The education of doctors and other health professionals is now firmly in the public domain, with the Royal Colleges, Health Commissions, NHS trusts, patients, and the GMC all having a stake in promoting lifelong learning. The emergence of clinical governance frameworks and the emphasis on clinical effectiveness mean that CPD is becoming increasingly important. Although it is voluntary now, it will inevitably become mandatory within the next few years.

The benefits of CPD have long been known in other professions, and are slowly being acknowledged by doctors. Through CPD we can achieve personal and professional growth, and develop, acquire, and refine the skills needed for new roles and responsibilities. The motivation for individual participation in CPD varies, but includes the following:

- improved care of patients

- intellectual challenge

- need for updating

- an anticipated need to provide evidence of competence

- compliance with employers' and professional bodies' requirements

- awareness of shortcomings

- approval of one's peers

- fear of litigation

- preparation for new roles.

\section{The College CPD scheme: why change to CPD?}

The concept of CPD is broad, with three main components: the knowledge, skills, and attributes that a doctor needs for professional practice. Continuing medical education (CME) is but one component of CPD and is traditionally viewed in terms of knowledge update-extremely important for doctors working in specialties where the knowledge base is expanding rapidly. However, there is a range of other academic, vocational, and professional activities that are essential for effective practice.

“....CPD for all staff is essential. Such a commitment will enable pathology services to evolve further and will enable pathology staff to communicate confidently with users and to manage a cost effective and high quality service." 2

The Royal College of Pathologists has embraced the concept of CPD and is responsible for setting educational standards and monitoring, facilitating, and evaluating CPD activities. By taking account of the changing context of practice, the College CPD scheme encourages members to identify learning needs in a wide range of areas, with credits awarded in a flexible way to reflect this. The College is developing ways to allocate more credits for active participation and achievements rather than just passive attendance at meetings. The introduction of a portfolio learning record in April 1999 will help members to keep a record of their activities that could form the basis of a learning plan within a system of job plan review and appraisal. The College also commissions and validates CPD materials and promotes and disseminates information on current learning methods, encouraging effective, individual, needs based education in all aspects of CPD.

Regular meetings between the CPD department and regional CPD chairs ensures effective communication and regional input into policy. The College also collaborates with key individuals and bodies to further the development of CPD, including the negotiation of funding and study leave to support CPD. It is imperative that the CPD scheme is subject to rigorous scrutiny and we are developing audit systems to maintain its integrity and quality. ". . ..certain colleges are broadening the scope of CME making their requirements more relevant to the contemporary NHS and the context of practice." ${ }^{3}$

\section{Roles and responsibilities of key groups in relation to CPD and clinical}

\section{governance}

INDIVIDUAL CONSULTANTS AND TRUSTS

Individuals are responsible for planning, documenting, and managing their own CPD (including their College CPD returns) as well as recognising obligations to their employer. Equally, employers (usually trusts) need to have a corporate commitment to CPD for consultants and other career grade specialists and should develop procedures which support CPD, provide developmental opportunities,
Accepted for publication 20 October 1998 
give positive encouragement and recognition, and assist with resources. ${ }^{4}$

Lifelong learning is an investment in quality and Trusts should value CPD as an integral part of quality improvement. ${ }^{1}$

\section{POSTGRADUATE CENTRES}

CPD programmes are best managed locally to meet both local service needs and those of individual consultants. Postgraduate centres should be a hub of information and educational activity and responsible for liaising with the relevant professional bodies that support CPD. Clinical tutors and postgraduate centre staff should work closely with specialty and college tutors to ensure that a range of high quality educational activities is organised and accessible for all consultants locally. These should include a range of multiprofessional educational events relevant to the needs of both individuals and the organisation, developed from audit, clinical effectiveness, and evidence based practice activities.

Trusts should support all consultants, senior scientists, and other career grade doctors (including associate specialists and academic staff with clinical sessions) in their CPD activities. For those activities that necessarily take place outside the workplace, consultants need protected time and study leave funding, with locums provided where possible. ${ }^{5}$

\section{THE COLLEGE}

In addition to the role of the CPD department already described, the RCPath intends to develop strong local CPD frameworks by encouraging more collaboration between college tutors, regional CPD chairs, postgraduate centres, and trust clinical tutors. The college tutor is the primary link between individual participants and the college CPD scheme. They should encourage pathologists to seek accreditation for a range of specialty based activities, including case discussions, journal clubs, and audit projects. Close liaison with local clinical tutors and postgraduate centres will help to identify and arrange accreditation for other local, non-specialist activities which may be relevant for CPD. These might include courses on information technology, appraisal, and interview skills. The college tutor can help and advise pathologists wishing to organise or set up local activities and advise on the implementation and use of the new learning portfolios.

The regional CPD advisors are responsible for encouraging the organisation and accreditation of regional CPD activities. They will have an overview of CPD in the region and through close liaison with college tutors and the regional CPD committee should compile a database of activity. Regular contact with the College provides important information about local issues, especially the allocation and funding of study leave.

\section{Identifying learning needs: how do we find out what we need to know?}

Doctors tend to go on courses to learn about things that they are already interested in and are not always objective about what their real learning needs are. There is also often a tension between the real and perceived educational needs of individuals and those of their employers. The introduction of job plan review, appraisal, and the development of learning plans can help to minimise this. Consultants actively participating in CPD should be encouraged to produce personal development plans and have access to and time for regular developmental peer review. Appraisal is already carried out in many trusts for management roles and in university departments, and is useful for identifying needs and resources. Junior doctor training at all levels (PRHO, SHO, and $\mathrm{SpR}$ ) now includes keeping records of learning and regular appraisal with educational supervisors. This process should continue into the career grade, replacing the educational supervisors with peer review. In this way, CPD takes account of individual specialist and trust needs, as well as NHS-wide issues. Table 1 shows some examples of ways in which we can identify the real gaps in our knowledge, development, and delivery of pathology services.

\section{What CPD activities should be recognised?}

Traditionally, CME activities involve attending lectures or courses for knowledge update, and these attract the bulk of CME credits in most schemes around the world. However, other learning methods may be more effective in terms of translation into improvements in practice. There are many activities (formal and informal) which form part of the CPD process

Table 1 Some methods of identifying learning needs

Peer review: within groups of specialists or departments, the learning needs of colleagues can be explored in relation to the business plan and future developments in the service.

Paired peer review: meet with a colleague and share outline learning plans, achievements, and areas for development; keep a learning record and compare outcomes.

Internal quality schemes: for example, in histopathology a review of random cases by peers to look at diagnosis, quality of slide staining, and adequacy of report. A multi-professional element can be introduced, where managers, secretaries, and MLSOs will have a view on the effectiveness of a pathologist's practice in terms of communication and other professional skills.

External quality schemes:

Individual performance, for example circulate slides, diagnostic problems or cases, give an opinion then circulate all diagnoses for discussion at a meeting and compare individuals' opinions with those of the group.

External review of departments, for example assessment of departmental performance and TQA in universities, accreditation for laboratories, College visits, and review for trainee posts can all highlight areas for learning and development, either at individual or at departmental level.

Learning portfolios: individuals can keep a record of learning activities, which, combined with reflective notes and peer review or discussion, can identify their needs in relation to local requirements.

Appraisal: linked with learning portfolios and job plan review.

Self assessment: the use of self assessment material in journals or using multimedia technology such as CD-ROMs, web assisted learning, etc.

Learning from mistakes: When a clinical mistake has been made this can be a trigger for individual reflection and identification of learning needs, as well as organisational review. For example: was the mistake due to lack of knowledge, overwork or stress, or lack of protocols or guidance? 
and which should be recognised. These include academic work such as reading, writing, publishing, teaching, and examining. Spending time in other departments or hospitals or going on sabbatical can be valuable learning experiences as well as powerful motivators for rekindling enthusiasm. Similarly, embarking on a diploma or degree course in a specialist or non-specialist area can change peoples' practice. Some other examples of learning activities that should be recognised for CPD credits are as follows.

\section{CLINICOPATHOLOGICAL CONFERENCES}

Discussion between clinicians and pathologists can be a valuable learning exercise. The process of arriving at a clinicopathological correlation can illuminate areas for reflection, improve practice, and identify learning needs.

WRITING AND REVISING SERVICE PROTOCOLS

The production of evidence based protocols is a useful way of learning and keeping up to date, especially when a review of the literature and audit results are involved.

MULTIPROFESSIONAL REVIEW

Pathology laboratories are subject to regular accreditation and review of all procedures and processes involving all staff, including MLSOs, pathologists, and administrative and clerical staff. The process of preparing for accreditation involves discussion and review of practice with input on a multiprofessional basis. The Australasian Royal College of Physicians formally recognises peer review as part of their CPD credit scheme. ${ }^{6}$

QUALITY ASSESSMENT SCHEMES

Diagnostic cyto/histopathology EQAs have an important educational function which relates to the intrinsic learning value of looking at slides or cases and to the quantitative feedback that is provided for each participant. ${ }^{7}$ Variations on this can be used for other specialties.

\section{Conclusions}

It is clear that in the context of professional self regulation and clinical governance, CPD is becoming increasingly important. Pathologists recognise their responsibility to keep up to date in their specialty and to acquire new expertise through a variety of means. College schemes need to be flexible enough to recognise a range of formal and informal activities for CPD and at the same time be rigorous about maintaining standards. Some trust attitudes are beginning to change, to encourage professional development and multiprofessional education as part of their core activity. However, although some trusts have coherent policies for financing study leave, for many individuals the important ingredients of time for reflection and access to relevant CPD frameworks locally are lacking. The continuous and ongoing nature of CPD means that defining criteria to measure its effectiveness remains difficult and we need more evidence about what educational interventions lead to improvements in practice and thus underpin College CPD schemes. ${ }^{8}$

The author is Director of CPD, Royal College of Pathologists, 2 Carlton House Terrace, London SW1 5AF.

1 A first class service: quality in the new NHS. London: NHSE Consultation document, 1998.

2 Strategic review of pathology services. London: Department of Health, 1995.

3 SCOPME recommendations for hospital consultant CPD and draft principles for all doctors and dentists, May 1998.

4 A framework for CPD systems. The Academy of Royal Medical Colleges, August 1998.

$5 \mathrm{du}$ Boulay C. Continuing medical education for pathologists: an evaluation of the RCPath's Wessex pilot scheme f Clin Pathol 1997;50:1022-6

6 Newble D. The maintenance of professional standards programme of the Royal Australasian College of Physicians. $\mathcal{F}$ $R$ Coll Physicians Lond 1996;30:252-6.

7 Recommendations for the development of histopathology external quality assessment schemes. Supplement to the Bulletin of the Royal College of Pathologists, June 1998.

8 du Boulay C. Audit of CME for pathologists: strategies and implications. F Clin Pathol 1996;49:100-1. 